TITLE:

\title{
Amygdala integrates emotional expression and gaze direction in response to dynamic facial expressions.
}

\section{AUTHOR(S):}

Sato, Wataru; Kochiyama, Takanori; Uono, Shota; Yoshikawa, Sakiko

\section{CITATION:}

Sato, Wataru ...[et al]. Amygdala integrates emotional expression and gaze direction in response to dynamic facial expressions.. Neurolmage 2010, 50(4): 1658-1665

\section{ISSUE DATE:}

2010-05-01

URL:

http://hdl.handle.net/2433/120951

\section{RIGHT:}

(C) 2010 Elsevier B.V.; この論文は著者最終稿です。内容が印刷版と異な ることがありますので、引用の際には出版社版をご確認ご利用くださ $\omega_{\circ}$ This is the Accepted Author Manuscript. Please cite only the published version. 


\section{Amygdala integrates emotional expression and gaze direction in}

\section{response to dynamic facial expressions}

Wataru Sato ${ }^{1}$, Takanori Kochiyama ${ }^{2}$, Shota Uono ${ }^{3}$, and Sakiko Yoshikawa $^{4}$

1 Department of Comparative Study of Cognitive Development (funded by Benesse Corporation), Primate Research Institute, Kyoto University, Inuyama, Aichi 484-8506, Japan.

2 Brain Activity Imaging Center, Advanced Telecommunications Research Institute International, 2-2-2 Hikaridai, Seika-cho, Soraku-gun, Kyoto 619-0288 Japan.

${ }^{3}$ Department of Cognitive Psychology in Education, Kyoto University, Yoshida-honmachi, Sakyo-ku, Kyoto 606-8501, Japan.

4 Kokoro Research Center, Kyoto University, Yoshida-honmachi, Sakyo-ku, Kyoto 606-8501, Japan.

\section{Corresponding author:}

Wataru Sato

Department of Comparative Study of Cognitive Development (Funded by Benesse Corporation), Primate Research Institute, Kyoto University, Aichi 484-8506, Japan

E-mail: sato@pri.kyoto-u.ac.jp 


\section{Abstract}

Neuroimaging studies suggest that the amygdala integrates emotional expression and gaze direction, but the findings are inconsistent. We hypothesized that the dynamic facial expressions, which are more salient stimuli than static facial expressions are, would reveal the integration of emotional expression and gaze direction in amygdala activity. To test this hypothesis, we presented dynamic and static facial expressions of anger and happiness looking toward and away from the subject, and we visualized brain activity during these presentations using functional magnetic resonance imaging (fMRI). After the image acquisition phase of the study, the subject's experienced emotion in response to each stimulus was investigated. The left amygdala showed an interaction between presentation condition and gaze direction, indicating higher activity in response to dynamic, but not static, expressions looking toward the subjects than to expressions looking away from them. This was true for both angry and happy expressions. This pattern corresponded with the intensity of emotional arousal. These results suggest that the amygdala integrates emotional expression and gaze direction in dynamic facial expressions and modulates emotional arousal.

\section{Keywords}

amygdala, dynamic facial expressions, experienced arousal, fMRI, gaze direction. 


\section{Introduction}

Facial expressions of emotion and gaze direction play an important role in daily face-to-face communication. Facial expressions indicate moment-to-moment changes in inner emotional states (Ekman and Friesen, 1975) and/or communicative intentions (Fridlund, 1997). Gaze direction provides information about the direction of attention and can be indicated by the eyes, face, or body orientation (Kobayashi and Kohshima, 2001). Ample evidence in social psychology indicates that these signals are tightly integrated (for review, see Ellsworth and Ludwig, 1972; Kleinke, 1986). For example, Kimble and Olszewski (1980) reported that when subjects were asked to look at people expressing negative and positive emotions either strongly or weakly, the subjects spent more time looking directly at the people expressing strong emotions. These findings led to the hypothesis that direct gaze intensifies emotional communication (Ellsworth, 1975).

Despite its psychological importance, the neural substrate mediating the integration of emotional expression and gaze direction is not known. Only a few studies have investigated this issue (Adams et al., 2003; Hadjikhani et al., 2008; Sato et al., 2004b), and the findings have been inconsistent. For example, in a functional magnetic resonance imaging (fMRI) study, Adams et al. (2003) presented the photos of angry expressions looking toward and away from the subjects. They found that angry expressions looking away from the subject elicited greater activation of the amygdala compared to angry expressions looking toward the subject. They supeculated that the amygdala processes 
ambiguity. In contrast, Sato et al. (2004b) first presented fullface neutral facial expressions followed by photos of angry and neutral expressions looking toward and away from the subjects. They found that angry expressions elicited greater activity in the amygdala when gaze was directed toward the subjects than when it was directed away from them. In addition, they found a positive relationship between amygdala activity and experienced emotion. Thus, they speculated that the amygdala plays a role in eliciting emotion. Together, these data suggest that the amygdala is involved in the integration of emotional expression and gaze direction, but the activating factors and psychological function of this role for the amygdala are not clear.

The varying findings of the previous studies (Adams et al., 2003; Sato et al., 2004b) may have resulted from differences in stimulus presentation. Whereas Adams et al. (2003) presented static facial-expression stimuli, Sato et al. (2004b) presented neutral and emotional expressions consecutively. It is reasonable to assume that this sequential presentation induced apparent motion (Kolers and von Grünau, 1976) and implemented pseudo-dynamic changes in facial expressions. Dynamic facial expressions of emotion are more ecologically valid and salient for emotional communication than static expressions. Dynamic facial expressions elicit higher emotional arousal than static facial expressions (Sato and Yoshikawa, 2007b), and several neuroimaging studies have shown that dynamic facial expressions evoke more activity in the amygdala than static facial expressions (LaBar et al., 2003; Pelphrey et al., 2007; Sato et al., 2004a). Based on these data, we 
hypothesized that the integration of emotional expression and gaze direction, specifically the emotion-intensifying effect of direct gaze (Ellsworth, 1975), would be more evident in amygdala activity in response to dynamic facial expressions than to static facial expressions.

To test our hypothesis, we measured brain activity using fMRI during the presentation of dynamic and static facial expressions either looking toward or away from the subject. We used angry and happy expressions. We chose these emotions for several reasons. First, the previous studies that used the angry expressions to investigate the integration of emotional expression and gaze direction reported inconsistent amygdala activation patterns (Adams et al., 2003; Sato et al., $2004 \mathrm{~b})$. Therefore, the amygdala activity in response to angry expressions with direct or averted gaze needs clarification. Second, anger and happiness represent negative and positive emotions, and the facial expressions of both emotions have been shown to activate the amygdala (e.g., Yang et al., 2002). Finally, behavioral studies have shown that these expressions interact with gaze direction (e.g., Kimble and Olszewski, 1980). We predicted that amygdala activity would show an interaction between the presentation condition and gaze direction. Specifically, we predicted that dynamic, but not static, expressions looking toward the subjects would induce greater amygdala activity than expressions looking away from the subjects under both the anger and happy conditions.

We investigated the psychological process corresponding to amygdala activity by presenting the same stimuli after the image 
acquisition and instructing the subjects to rate their experienced emotion using valence and arousal scales. Valence represents the qualitative component of emotion (i.e., positive or negative), and arousal reflects the intensity of the emotion (Lang et al., 1998). We investigated subjective emotion because several neuroimaging studies have revealed that amygdala activity was associated with subjective emotional arousal/intensity (Anderson et al., 2003; Canli et al., 2000; Phan et al., 2003; Sato et al., 2004b, in press; Small et al., 2003; Zald and Pardo, 1997; Zald et al., 1998). Based on these data, we predicted that emotional arousal would be reflected in the interaction between the presentation condition and gaze direction, with corresponding amygdala activity.

\section{Material and methods}

\section{Subjects}

Twenty-one volunteers (10 women and 11 men; mean \pm SD age, 22.3 \pm 2.0 years $)$ participated in the experiment. All subjects were right-handed, as assessed by the Edinburgh Handedness Inventory (Oldfield, 1971), and had normal or corrected-to-normal visual acuity. All subjects gave informed consent, and the study was conducted in accordance with the institutional ethical provisions and the Declaration of Helsinki.

\section{Experimental design}

The experiment had a within-subject three-factorial design, with 
presentation condition (dynamic, static), emotional expression (angry, happy), and gaze direction (toward, away).

\section{Stimuli}

Angry and happy facial expressions of four women and four men were presented as video clips. These stimuli were selected from our video database of facial expressions of emotion that includes more than 50 Japanese models. None of the faces was familiar to any of the subjects. The stimuli looking toward and away from the subject were simultaneously recorded by the video cameras in front of the model and at a $30^{\circ}$ angle to the model's left. The stimulus model looked straight ahead for every condition.

The dynamic expression stimuli were composed of 38 frames ranging from neutral to emotional in expression. Each frame was shown for $40 \mathrm{~ms}$, and each clip was presented for $1520 \mathrm{~ms}$. The frames showing the peak of expression under the dynamic condition were used for the static expression stimuli and were also presented for $1520 \mathrm{~ms}$. The stimuli subtended a visual angle of about $15^{\circ}$ vertical $\times 12^{\circ}$ horizontal. An example of the stimulus sequence is shown in Fig. 1.

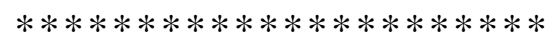

Fig. 1

$* * * * * * * * * * * * * * * * * * * * * *$

To ensure the validity of stimuli, we conducted a preliminary rating experiment using the stimuli for the dynamic gaze toward condition with 14 subjects who did not take part in this experiment. The subjects were instructed to evaluate each stimulus for the intensity 
of recognized emotion using a 7-point scale for six basic emotions (angry, disgust, fear, happiness, sadness, surprise). The results confirmed that all the stimuli significantly displayed the target emotions more strongly than other basic emotions (multiple comparisons with the Ryan method, $P$ s $<0.05)$. In addition, a coder trained in the use of the Facial Action Coding System (FACS; Ekman and Friesen, 1978) evaluated the facial movements of the stimuli, and the resultant FACS data were subjected to the Facial Action Coding System Affect Interpretation Dictionary (FACSAID; Ekman et al., 1998). The results confirmed that the patterns of facial muscular changes shown in these stimuli were appropriate as typical emotional facial expressions. The speed of these stimuli has also been shown to sufficiently represent natural changes in the dynamic facial expressions of anger and happiness (Sato and Yoshikawa, 2004). These stimuli have been used in previous behavioral studies and shown to elicit appropriate behavioral (Sato and Yoshikawa, 2007a) and subjective (Sato and Yoshikawa, 2007b) responses. Taken together, these data support the validity of the present stimuli.

\section{Presentation apparatus}

The events were controlled by Presentation Software version 10.0 (Neurobehavioral Systems, Albany, CA, USA) implemented on a computer using Microsoft Windows. The stimuli were projected from a liquid crystal projector (DLA-G150CL; Victor Electronics, Brussels, Belgium) to a mirror that was positioned in a scanner in front of the 
subjects.

\section{Procedure}

Each subject completed the experimental scan twice. Each scan consisted of sixteen 24-s epochs with sixteen 24-s rest periods (a blank screen) in between. Each of the eight stimulus conditions was presented in different epochs within each scan. The order of epochs within each run was pseudorandomized. The order of stimuli within each epoch was randomized.

Each epoch consisted of eight trials. In each trial, a fixation point (a small gray "+" on a white background the same size as the stimulus) was presented at the center of the screen for $1480 \mathrm{~ms}$. The stimulus was then presented for $1520 \mathrm{~ms}$. The subjects were instructed to look at the center of the screen until the face disappeared and then asked to indicate the gender of the face by pressing one of two buttons with their forefingers. This task ensured that the subjects attended to the stimuli without paying particular attention to the emotional expression and gaze direction. Post-hoc debriefing confirmed that the subjects were not aware that the purpose of the experiment was to investigate emotional variables.

After MRI image acquisition, the stimuli were again randomly presented to the subjects, who evaluated each stimulus for emotional valence and arousal using the affect grid (Russell et al., 1989), which graphically assesses the two dimensions of pleasure and arousal on a 9-point scale. 


\section{MRI acquisition}

Image scanning was performed on a $3 \mathrm{~T}$ scanning system (MAGNETOM Trio A, Tim System; Siemens, Malvern, PA, USA) using a 12-channel head coil. A forehead pad was used to stabilize the head position. The functional images consisted of 50 consecutive slices parallel to the anterior and posterior commissure plane, covering the whole brain. A T2*-weighted gradient-echo echo-planar imaging sequence was used with the following parameters: repetition time $(\mathrm{TR})=$ $3000 ;$ echo time $(\mathrm{TE})=30 \mathrm{~ms} ;$ flip angle $=90^{\circ} ;$ matrix size $=64 \times 64$; voxel size $=3 \times 3 \times 3 \mathrm{~mm}$. After the acquisition of functional images, a T1-weighted high-resolution anatomical image was obtained using magnetization prepared rapid-acquisition gradient-echo (MP-RAGE) sequence $\left(\mathrm{TR}=2250 \mathrm{~ms} ; \mathrm{TE}=3.06 \mathrm{~ms} ;\right.$ flip angle $=9^{\circ}$; field of view $=$ $256 \times 256 \mathrm{~mm}$; voxel size $=1 \times 1 \times 1 \mathrm{~mm})$.

\section{Behavioral data analysis}

The rating data for psychological ratings after image acquisition were analyzed using a 2 (presentation condition: dynamic, static) $\times 2$ (emotional expression: angry, neutral) $\times 2$ (gaze direction: toward, away) repeated-measures analysis of variance (ANOVA). Simple effect analyses were conducted for significant interactions. Accuracy and reaction time data for gender classification were analyzed with ANOVAs for the same factors. $P$ values of $<0.05$ were deemed to be statistically 
significant.

\section{Image analysis}

Image and statistical analyses were performed using the statistical parametric mapping package SPM5 (http://www.fil.ion.ucl.ac.uk/spm) implemented in MATLAB Version 7 (Mathworks Inc., Natick, MA, USA). Functional images of each run were realigned using the first scan as a reference to correct for head movements. Data from all 21 subjects showed a small motion correction $(<2 \mathrm{~mm})$. The $\mathrm{T} 1$ anatomical image was preprocessed by intensity inhomogeneity correction. Then, T1 anatomical images were coregistered to the first scan of the functional images. Following this, the coregistered T1 anatomical image was normalized to a standard T1 template image as defined by the Montreal Neurological Institute (MNI) involving linear and nonlinear three-dimensional transformations (Ashburner and Friston, 1999; Friston et al., 1995a). The parameters from this normalization process were then applied to each of the functional images. Finally, these spatial normalized functional images were resampled to a voxel size of $2 \times 2 \times$ 2 and smoothed with an isotopic Gaussian kernel of $8-\mathrm{mm}$ full-width at half-maximum to compensate for anatomic variability among subjects.

We used random effects analyses (Holmes and Friston, 1998) to search for significantly activated voxels that displayed interesting effects. First, we performed a single-subject analysis (Friston et al., 1995b; Worsley and Friston, 1995). The task-related neural activities for each condition were modeled with a boxcar function, convoluted with a 
canonical hemodynamic response function. We used a high-pass filter composed of a discrete cosine basis function with a cutoff period of 128 to eliminate the artifactual low-frequency trend. Serial autocorrelation, assuming a first-order autoregressive model, was estimated from the pooled active voxels with a restricted maximum likelihood (ReML) procedure and was used to whiten the data and the design matrix (Friston et al., 2002).

Planned contrast was thereafter performed for the interaction, which was between dynamic versus static presentation conditions and toward versus away directions, based on our a priori hypothesis. For this analysis, a contrast image was generated for each subject and then entered into a one-sample $t$-test to create a random effect $\operatorname{SPM}\{T\}$. Other main effects and interactions were also tested using two-tailed $\operatorname{SPM}\{T\}$. Significantly activated voxels were identified if they reached the extent threshold of $P<0.05$ corrected for multiple comparisons, with height threshold of $P<0.001$ (uncorrected). For the amygdala, about which we had prior anatomical hypotheses, a small volume correction (SVC) with an anatomical mask was used (Worsley et al., 1998). The search region in the amygdala was determined by tracing strict anatomical borders defined by the cytoarchitectonic map derived from the data of human postmortem brains using the Anatomy Toolbox Version 1.5 (Amunts et al., 2005; Eickhoff et al., 2005). Other areas were corrected for the entire brain volume.

To scrutinize the activation patterns of the amygdala, we further conducted region of interest (ROI) analyses. Based on the results of the 
interaction contrast between presentation condition and gaze direction, the activity of the left amygdala was analyzed. The percentage signal change at the coordinates of the group maximum in the random effects analysis $(x-18, y-10, z-10)$ was extracted in the single-subject analysis for each condition (regression coefficient) against the baseline (i.e. the coefficient of the session constant term) using Marsbar Toolbox Version 0.42 (http://marsbar.sourceforge.net/) (Brett et al., 2002). Then, the data were analyzed using a 2 (presentation condition) $\times 2$ (emotional expression $) \times 2$ (gaze direction) repeated-measures ANOVA. Simple effect analyses were conducted for significant interactions. $P$ values of $<$ 0.05 were considered statistically significant.

\section{Results}

Psychological rating

\section{Valence.}

With regard to experienced valence (Fig. 2, left), the three-way ANOVA showed a significant main effect of emotional expression, with higher negative valence ratings for angry than for happy expressions $\left(F_{1,20}=68.43, P<0.001\right)$. The two-way interaction between presentation condition and emotional expression was also significant $\left(F_{1,20}=5.08, P\right.$ $<0.05)$. The emotional expression $\times$ gaze direction interaction showed a nonsignificant trend $\left(F_{1,20}=4.30, P<0.1\right.$, respectively). No other main effects or interactions were significant $(P \mathrm{~s}>0.1)$.

Fig. 2 
Neuroimage 14

$* * * * * * * * * * * * * * * * * * * * * *$

Simple effect analyses for the presentation condition $\times$ emotional expression interaction indicated that dynamic presentation elicited more positive valence ratings than the static presentation for happy expressions $\left(F_{1,40}=7.84, P<0.01\right)$, whereas the presentation condition showed no significant effect for angry expressions $\left(F_{1,40}=0.02, P>\right.$ $0.1)$.

Arousal.

For experienced arousal (Fig. 2, right), the ANOVA showed a significant main effect of presentation condition, with higher arousal ratings for dynamic than for static presentations $\left(F_{1,20}=7.63, P<0.05\right)$. More importantly, the results showed a significant interaction between the presentation condition and gaze direction $\left(F_{1,20}=10.05, P<0.005\right)$. The two-way interaction of presentation condition $\times$ emotional expression and the three-way interaction of presentation condition $x$ emotional expression $\times$ gaze direction showed trends toward significance $\left(F_{1,20}=3.51\right.$ and $3.78, P$ s $\left.<0.1\right)$. No other main effects or interactions were significant $(P \mathrm{~s}>0.1)$.

Simple effect analyses for the presentation condition $\times$ gaze direction interaction showed that expressions looking toward the subject elicited greater arousal than expressions looking away from the subject under the dynamic presentation condition $\left(F_{1,40}=11.99, P<0.005\right)$, but not under the static condition $\left(F_{1,40}=1.61, P>0.1\right)$. 


\section{Gender classification}

Performance on the gender classification task was close to perfect (correct identification rate $=98.2 \%)$. No significant main effects or interactions were found for accuracy or reaction times (three-way ANOVAs, $\left.F \mathrm{~s}_{1,20}<2.86, P \mathrm{~s}>0.1\right)$.

\section{Brain activity}

Interaction between presentation condition and gaze direction.

A planned contrast of the interaction between presentation condition and gaze direction revealed significant left amygdala activation (Fig. 3, Table 1). The right amygdala also showed activation associated with this interaction, but it failed to reach significance of extent threshold ( $x-24$, $\left.\mathrm{y}-18, \mathrm{z}-4 ; T_{20}=4.87\right)$, suggesting that the amygdala activation was not clearly lateralized.

$$
* * * * * * * * * * * * * * * * * * * * * *
$$

Fig. 3 and Table 1

$* * * * * * * * * * * * * * * * * * * * * *$

To further analyze the reflection of the interaction between presentation condition and gaze direction in amygdala activity, we conducted ROI analysis. The three-way ANOVA for the left amygdala ROI data showed a significant main effect of presentation condition, with higher activity for dynamic than for static presentations $\left(F_{1,20}=\right.$ 5.71, $P<0.05)$. More importantly, the interaction between presentation condition and gaze direction was significant $\left(F_{1,20}=12.33, P<0.005\right)$. No other main effects or interactions were significant $(P$ s $>0.1)$. Simple 
effect analyses for the interaction revealed that expressions directed toward the subject elicited higher amygdala activity than expressions directed away from the subject under the dynamic presentation condition $\left(F_{1,40}=7.00, P<0.05\right)$, and the opposite pattern reached marginal significance under the static presentation condition $\left(F_{1,40}=3.40, P<\right.$ $0.1)$.

This contrast in the SPM analysis detected significant activation in areas other than the amygdala, including the right lingual gyrus and right fusiform gyrus (Table 1).

Other effects.

Table 1 shows the results of other main effects and interactions. For the main effect of presentation condition, significant activation clusters were found in the bilateral lateral posterior visual cortices, including middle temporal gyri, indicating higher activity for dynamic presentations than for static presentations. This contrast also detected significant activation in the right amygdala, indicating higher activity for dynamic than for static presentations, although it failed to reach significance of extent threshold $\left(\mathrm{x}-24, \mathrm{y}-14, \mathrm{z}-6 ; T_{20}=4.54\right)$. No significant activation was found for the main effects of emotional expression and gaze direction. For the interaction between presentation condition and emotional expression, significant activation was found in the right cerebellum. For the interaction between emotional expression and gaze direction, significant activation was found in the bilateral cerebellum and right ventral occipital cortices. No significant activation 
was found in the three-way interaction.

\section{Discussion}

The results of the psychological ratings revealed that gaze direction modulates valence and arousal in response to facial expressions. Dynamic stimuli elicited greater arousal when facial expressions were directed toward the subject than when they were looking away. This was true for both angry and happy expressions. Gaze direction had less impact on emotional arousal when the facial expressions were static. Valence and arousal indicate the quality and intensity of emotions (Lang et al., 1998), and these results suggest that dynamic expressions with direct gaze enhance the subjective emotional reaction. This is consistent with the findings of previous social psychology studies that direct gaze facilitated emotional communication when realistic situations were used (Ellsworth and Ludwig, 1972). Our results are also in line with a recent report showing that direct gaze evoked a greater autonomic reaction than averted gaze in live, but not static, face stimuli (Hietanen et al., 2008). In addition, our valence rating results revealed that the gaze direction can modulate qualitative emotional reactions.

In accordance with our prediction, amygdala activity revealed an interaction between presentation condition and gaze direction. The simple main effect in the ROI analysis indicated that when dynamic stimuli were used, facial expressions directed toward the subject elicited more amygdala activity than those looking away. In contrast, direct gaze did not have a facilitative effect on amygdala activity when 
static stimuli were presented. Our results are consistent with those of Sato et al. (2004b), who used pseudo-dynamic presentations and found that angry expressions directed toward the subject elicited more amygdala activity and a greater emotional response than stimtli looking away. In addition, our results are in line with previous reports showing that amygdala activity was associated with emotional arousal/intensity using olfactory and gustatory stimuli (Anderson et al., 2003; Small et al., 2003; Zald and Pardo, 1997; Zald et al., 1998), scene stimuli (Canli et al., 2000; Phan et al., 2003), and facial expression stimuli (Sato et al., 2004b; Sato et al., in press). Taken together these results suggest that the amygdala integrates emotional expression and gaze direction in response to dynamic facial expressions, and plays a role in eliciting emotional responses.

The patterns of amygdala activity were similar for both angry and happy expressions and agree with previous reports of amygdala activity in response to emotional facial expressions of anger and happiness (e.g., Yang et al., 2002; for a review, see Sergerie et al., 2008).

In contrast to the dynamic presentation condition, the results for the static presentation condition showed a tendency toward lower amygdala activity in response to facial expressions looking toward the subjects than in response to those looking away. A previous study reported similar results with static angry expressions (Adams et al., 2003). Our findings suggest that the inconsistent results from previous studies may be accounted for by the difference between static (Adams et al., 2003) and pseudo-dynamic (Sato et al., 2004b) stimulus presentations. 
Regarding their findings, Adams et al. (2003) speculated that the observed amygdala activity might reflect the processing of ambiguity. It has been proposed that the amygdala may be involved in processing ambiguity as well as emotion (Whalen, 1998). This idea has been supported by some previous neuroimaging studies, which reported that the amygdala was active when subjects were viewing bizarre scenes (Hamann et al., 2002) or making decisions under ambiguous conditions (Hsu et al., 2005). In the present study, the emotion-intensifying effect of direct gaze (Ellsworth, 1975) was not evident under the static condition, probably because static expressions appear less realistic and have less emotional impact compared to dynamic expressions (Sato and Yoshikawa, 2007b). The lack of emotional impact generated by the direct-gaze condition might have accentuated the ambiguity of the averted-gaze condition in which the stimulus persons displayed emotional expressions to nonexistent targets. However, emotional expressions are dynamic in real life. Our results suggest that the amygdala is involved in the integration of emotional expression and gaze direction in daily dynamic social interactions, specifically in the intensified emotional arousal associated with direct gaze.

Our results also revealed that compared to the static stimuli, the dynamic stimuli elicited increased activity in the temporal cortices, including the middle temporal gyrus. These results are consistent with previous studies that compared brain activity in response to dynamic and static emotional facial expressions (Kilts et al., 2003; LaBar et al., 2003; Pelphrey et al., 2007; Sato et al., 2004a). Our analysis included 
cerebellar activity related to the interaction of presentation condition $x$ emotional expression and emotional expression $\times$ gaze direction.

Although the functional significance of the cerebellum is not clear, several previous neuroimaging studies have reported cerebellar activity in response to facial expressions (e.g., Sato et al., 2004a). Our results suggest that the cerebellum integrates facial expressions and gaze direction and may play a complex role in face-to-face communication.

The present study has some limitations. First, although our results suggest that amygdala activity is related to subjective emotional arousal in the integral processing of emotional expression and gaze direction, it is possible that amygdala activity is related to another psychological/physiological response. A previous fMRI study (Williams et al., 2001) revealed that amygdala activity in response to emotional facial expressions was related to electrodermal activity, which was shown to covary with subjective emotional arousal (Lang et al., 1993). Furthermore, several psychological studies (Adams and Kleck, 2003, 2005; Bindemann et al., 2007; Sander et al., 2006) have demonstrated an interaction between gaze direction and emotional expression on emotion recognition, which is related to subjective emotion (Richards et al., 2002). Future studies investigating these responses may elucidate the role of the amygdala in the integrative processing of emotional expression and gaze direction.

Second, we tested only angry and happy facial expressions. Hence, the effect of gaze direction on other emotions remains unknown. Some previous behavioral studies have shown that the gaze direction has 
different effects on emotion recognition depending on facial emotion; averted gaze facilitates recognition of emotion in the cases of fearful or sad expressions (Adams and Kleck, 2003, 2005; cf. Bindemann et al., 2007). These data suggest that the use of other emotions would be promising to further investigate this issue. However, it must be noted that two previous neuroimaging studies investigated the integrative processing of emotional expression and gaze direction using fearful expressions (Adams et al., 2003; Hadjikhani et al., 2008), and they reported inconsistent findings regarding amygdala activity. Because both of these studies used static stimuli, based on the present results, we speculate that dynamic stimuli may more clearly reveal the integration of emotional expression and gaze direction in amygdala activity for facial expressions of fear and other emotions.

Third, the temporal sequence of the dynamic changes in emotional expression and gaze direction might have influenced the results. Our stimuli under the dynamic condition identified the gaze direction at the beginning and thereafter changed emotional expressions. A recent behavioral study (Graham and Labar, 2007) found that when the stimuli included simultaneous changes in facial expression and gaze direction and the emotional expression was clear, as in the present study, gaze direction did not modulate the processing of emotional expressions. These results suggest that the sequential stimulus presentations in the present study might have enhanced the interaction effect of gaze direction on the processing of emotional expressions compared to a situation in which these two signals are presented simultaneously. More 
studies are warranted to systematically manipulate the temporal order of emotional expression and gaze direction. However, because a previous study (Sato et al., 2004b), in which the stimuli changed facial expression and gaze direction simultaneously, showed patterns in the amygdala activity similar to the present results, one can speculate that the temporal sequence of dynamic changes in emotional expression and gaze direction might have little impact on amygdala activity.

Finally, only head direction was changed to depict gaze direction. This had the advantage of complete simultaneous filming, but gaze direction can also be indicated by eye and body position. Future studies are necessary to test the effect of those modalities. However, evidence exists suggesting that the recognition of and response to angry and happy expressions were similar for eye and head direction (Sato and Yoshikawa, submitted).

\section{Conclusion}

Our results showed that amygdala activity was associated with an interaction between presentation condition and gaze direction, indicating that both angry and happy dynamic facial expressions elicited more activity in response to a gaze directed toward the subject than to a gaze that looked away. Furthermore, the amygdala activity corresponded to experienced emotional arousal. These results suggest that the amygdala is involved in the integration of emotional facial expression and gaze direction, and the response corresponds to emotional arousal. 


\section{References}

Adams, R.B., Kleck, R.E., 2003. Perceived gaze direction and the processing of facial displays of emotion. Psychol. Sci. 14, $644-647$.

Adams, R.B., Kleck, R.E., 2005. Effects of direct and averted gaze on the perception of facially communicated emotion. Emotion 5, $3-11$.

Adams, R.B., Gordon, H.L., Baird, A.A., Ambady, N., Kleck, R.E., 2003. Effects of gaze on amygdala sensitivity to anger and fear faces. Science $300,1536$.

Amunts, K., Kedo, O., Kindler, M., Pieperhoff, P., Mohlberg, H., Shah, N.J., Habel, U., Schneider, F., Zilles, K., 2005. Cytoarchitectonic mapping of the human amygdala, hippocampal region and entorhinal cortex: intersubject variability and probability maps. Anat. Embryol. 210, 343-352.

Anderson, A.K., Christoff, K., Stappen, I., Panitz, D., Ghahremani, D.G., Glover, G., Gabrieli, J.D., Sobel, N., 2003. Dissociated neural representations of intensity and valence in human olfaction. Nat. Neurosci. 6, 196-202.

Ashburner, J., Friston. K., 1999. Nonlinear spatial normalization using basis functions. Hum. Brain Mapp. 7, 254-266.

Bindemann, M., Burton, M.A., Langton S.R.H., 2007. How do eye gaze and facial expression interact? Vis. Cogn. 16, 708-733.

Brett, M., Anton, J.L., Valabregue, R., Poline, J.B., 2002. Region of interest analysis using an SPM toolbox. Paper presented at the 
Eighth International Conference on Functional Mapping of the Human Brain. Sendai, Japan, June, 2002.

Canli, T., Zhao, Z., Brewer, J., Gabrieli, J.D., Cahill, L., 2000.

Event-related activation in the human amygdala associates with later memory for individual emotional experience. J. Neurosci. 20, RC99.

Eickhoff, S.B., Stephan, K.E., Mohlberg, H., Grefkes, C., Fink, G.R., Amunts, K., Zilles, K., 2005. A new SPM toolbox for combining probabilistic cytoarchitectonic maps and functional imaging data. NeuroImage 25, 1325-1335.

Ekman, P., Friesen, W.V., 1975. Unmasking the Face: A Guide to Recognizing Emotions from Facial Clues. Prentice-Hall, Englewood Cliffs.

Ekman, P., Friesen, W.V., 1978. Facial Action Coding System. Consulting Psychologist Press, Palo Alto.

Ekman, P., Hager, J., Irwin, W., Rosenberg, E., 1998. Facial Action Coding System Affect Information Database (FACSAID). http://www.face-and-emotion.com/dataface/facsaid/description. jsp.

Ellsworth, P.C., 1975. Direct gaze as a social stimulus: the example of aggression. In: Pliner, P., Krames, L., Alloway, T. (Eds.), Nonverbal Communication of Aggression. Plenum Press, New York, pp. 53-75.

Ellsworth, P.C., Ludwig, L.M., 1972. Visual behavior in social interaction. J. Commun. 22, 375-403. 
Fridlund, A., 1997. The new ethology of human facial expressions. In: Russell, J.A., Fernandez-Dols, J.M. (Eds.), The Psychology of Facial Expression. Cambridge University Press, New York, pp. $103-129$.

Friston, K.J., Ashburner, J., Poline, J.B., Frith, C.D., Heather, J.D., Frackowiak, R.S.J., 1995a. Spatial registration and normalization of images. Hum. Brain Mapp. 2, 165-189.

Friston, K.J., Holmes, A.P., Poline, J.B., Grasby, P.J., Williams, S.C., Frackowiak, R.S.J., Turner, R., 1995b. Analysis of fMRI time-series revisited. NeuroImage 2, 45-53.

Friston, K.J., Penny, W., Kiebel, S., Hinton, G., Ashburner, J., 2002. Classical and Bayesian inference in neuroimaging: theory. NeuroImage 16, 465-483.

Graham, R., LaBar, K.S., 2007. Garner interference reveals dependencies between emotional expression and gaze in face perception. Emotion 7, 296-313.

Hadjikhani, N., Hoge, R., Snyder, J., De Gelder, B., 2008. Pointing with the eyes: the role of gaze in communicating danger. Brain Cogn. 68, $1-8$.

Hamann, S.B., Ely, T.D., Hoffman, J.M., Kilts, C.D., 2002. Ecstasy and agony: activation of the human amygdala in positive and negative emotion. Psychol. Sci. 13, 135-141.

Hietanen, J.K., Leppänen, J.M., Peltola, M.J., Linna-aho, K., Ruuhiala, H.J., 2008. Seeing direct and averted gaze activates the approach-avoidance motivational brain systems. Neuropsychologia 
$48,2423-2430$.

Holmes, A.P., Friston, K.J., 1998. Generalisability, random effects and population inference. NeuroImage 7, S754.

Hsu, M., Bhatt, M., Adolphs, R., Tranel, D., Camerer, C.F., 2005. Neural systems responding to degrees of uncertainty in human decision-making. Science, 310, 1680-1683.

Kilts, C.D., Egan, G., Gideon, D.A., Ely, T.D., Hoffman, J.M., 2003. Dissociable neural pathways are involved in the recognition of emotion in static and dynamic facial expressions. NeuroImage 18, $156-168$.

Kimble, C.E., Olszewski, D.A., 1980. Gaze and emotional expression: the effects of message positivity-negativity and emotional intensity. J. Res. Pers. 14, 60-69.

Kleinke, C.L., 1986. Gaze and eye contact: a research review. Psychol. Bull. 100, 78-100.

Kobayashi, H., Kohshima, S., 2001. Evolution of the human eye as a device for communication. In: Matsuzawa T. (Ed.), Primate Origins of Human Cognition and Behavior. New York, Springer-Verlag. Pp. 383-401.

Kolers, P.A., von Grünau, M., 1976. Shape and color in apparent motion. Vision Res. 16, 329-335.

LaBar, K.S., Crupain, M.J., Voyvodic, J.T., McCarthy, G., 2003. Dynamic perception of facial affect and identity in the human brain. Cereb. Cortex 13,1023-1033.

Lang, P.J., Greenwald, M.K., Bradley, M.M., Hamm, A.O., 1993. 
Looking at pictures: affective, facial, visceral, and behavioral reactions. Psychophysiology 30, 261-273.

Lang, P.J., Bradley, M.M., Cuthbert, B.N., 1998. Emotion, motivation, and anxiety: brain mechanisms and psychophysiology. Biol. Psychiatr. 44, 1248-1263.

Oldfield, R.C., 1971. The assessment and analysis of handedness: the Edinburgh inventory. Neuropsychologia 9, 97-113.

Pelphrey, K.A., Morris, J.P., McCarthy, G., Labar K.S., 2007. Perception of dynamic changes in facial affect and identity in autism. Soc. Cogn. Affect. Neurosci. 2, 140-149.

Phan, K.L., Taylor, S.F., Welsh, R.C., Decker, L.R., Noll, D.C., Nichols, T.E., Britton, J.C., Liberzon, I., 2003. Activation of the medial prefrontal cortex and extended amygdala by individual ratings of emotional arousal: a fMRI study. Biol. Psychiatry 53, $211-215$.

Richards, A., French, C.C., Calder, A.J., Webb, B., Fox, R., Young, A.W., 2002. Anxiety-related bias in the classification of emotionally ambiguous facial expressions. Emotion 2, 273-287.

Russell, J.A., Weiss, A., Mendelsohn, G.A., 1989. Affect grid: a single-item scale of pleasure and arousal. J. Pers. Soc. Psychol. $57,493-502$.

Sander, D., Grandjean, D., Kaiser, S., Wehrle, T., Scherer, K.R., 2006. Interaction effects of perceived gaze direction and dynamic facial expression: evidence for appraisal theories of emotion. Eur. J. Cogn. Psychol. 19, 470-480. 
Sato, W., Yoshikawa, S., 2004. The dynamic aspects of emotional facial expressions. Cogn. Emo. 18, 701-710.

Sato, W., Yoshikawa, S., 2007a. Spontaneous facial mimicry in response to dynamic facial expressions. Cognition 104, 1-18.

Sato, W., Yoshikawa, S., 2007b. Enhanced experience of emotional arousal in response to dynamic facial expressions. J. Nonverbal Behav. 31, 119-135.

Sato, W., Kochiyama, T., Yoshikawa, S., Naito, E., Matsumura, M., 2004a. Enhanced neural activity in response to dynamic facial expressions of emotion: an fMRI study. Cogn. Brain Res. 20, $81-91$.

Sato, W., Yoshikawa, S., Kochiyama, T., Matsumura, M., 2004b. The amygdala processes the emotional significance of facial expressions: an fMRI investigation using the interaction between expression and face direction. Neuroimage 22, 1006-1013.

Sato, W., Kochiyama, T., Yoshikawa, S., in press. Amygdala activity in response to forward versus backward dynamic facial expressions. Brain Res.

Sergerie, K., Chochol, C., Armony, J.L., 2008. The role of the amygdala in emotional processing: a quantitative meta-analysis of functional neuroimaging studies. Neurosci. Biobehav. Rev. 32, $811-830$.

Small, D.M., Gregory, M.D., Mak, Y.E., Gitelman, D., Mesulam, M.M., Parrish, T., 2003. Dissociation of neural representation of intensity and affective valuation in human gustation. Neuron 39, 
$701-711$

Whalen, P.J., 1998. Fear, vigilance, and ambiguity: initial neuroimaging studies of the human amygdala. Curr. Dir. Psychol. Sci. 7, 177-188.

Williams, L.M., Phillips, M.L., Brammer, M.J., Skerrett, D., Lagopoulos, J., Rennie, C., Bahramali, H., Olivieri, G., David, A.S., Peduto, A., Gordon, E., 2001. Arousal dissociates amygdala and hippocampal fear responses: evidence from simultaneous fMRI and skin conductance recording. NeuroImage 14, 1070-1079.

Worsley, K.J., Friston, K.J., 1995. Analysis of fMRI time-series revisited--again. Neuroimage 2, 173-181.

Worsley, K.J., Marrett, S., Neelin, P., Vandal, A.C., Friston, K.J., Evans, A.C., 1998. A unified statistical approach for determining significant signals in images of cerebral activation. Hum. Brain Mapp. 4, 58-73.

Yang, T.T., Menon, V., Eliez, S., Blasey, C., White, C.D., Reid, A.J., Gotlib, I.H., Reiss, A.L., 2002. Amygdalar activation associated with positive and negative facial expressions. NeuroReport 13, $1737-1741$.

Zald, D.H., Pardo, J.V., 1997. Emotion, olfaction, and the human amygdala: amygdala activation during aversive olfactory stimulation. Proc. Natl. Acad. Sci. U. S. A. 94, 4119-4124.

Zald, D.H., Lee, J.T., Fluegel, K.W., Pardo, J.V., 1998. Aversive gustatory stimulation activates limbic circuits in humans. Brain 121, 1143-1154. 


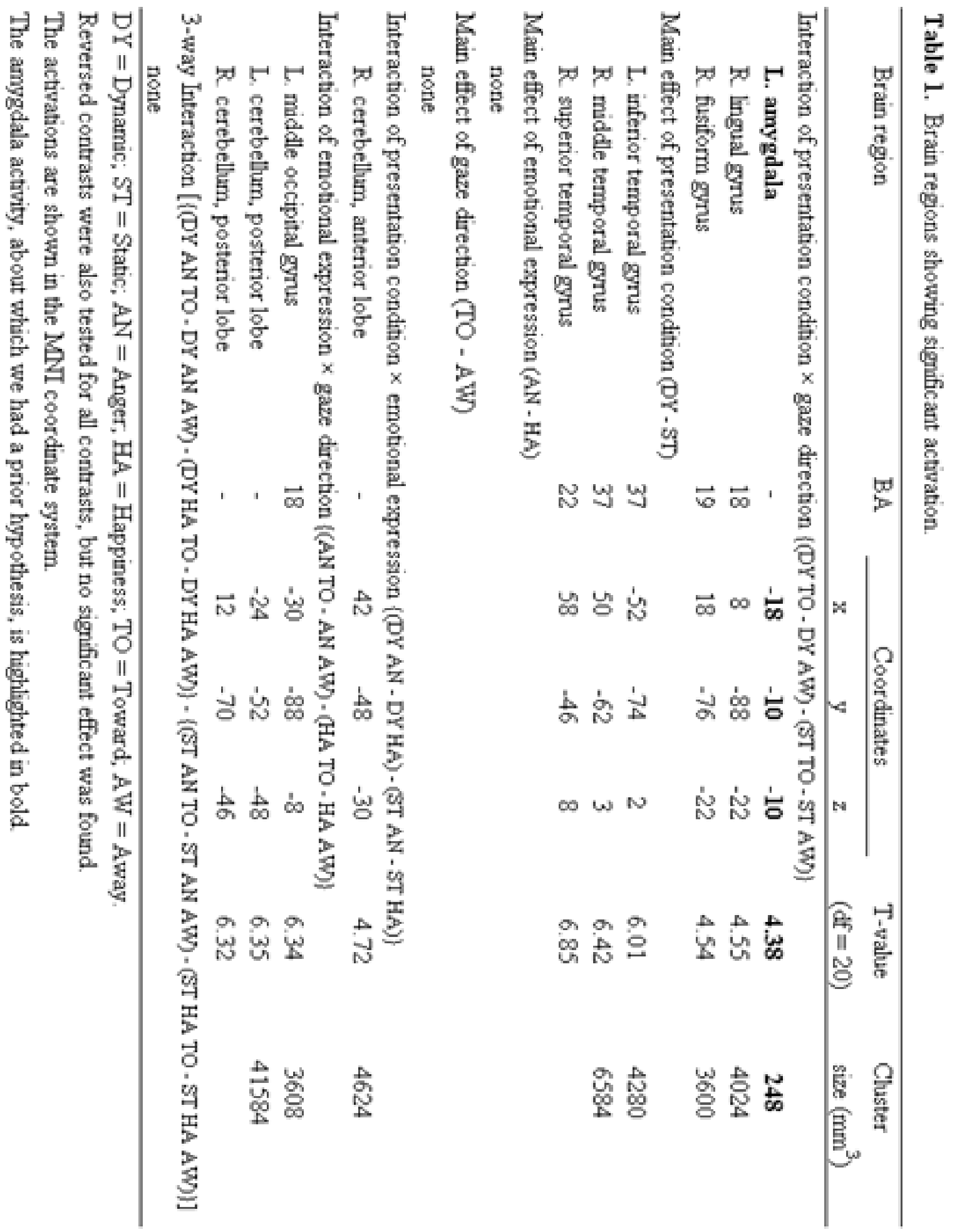


Neuroimage 31

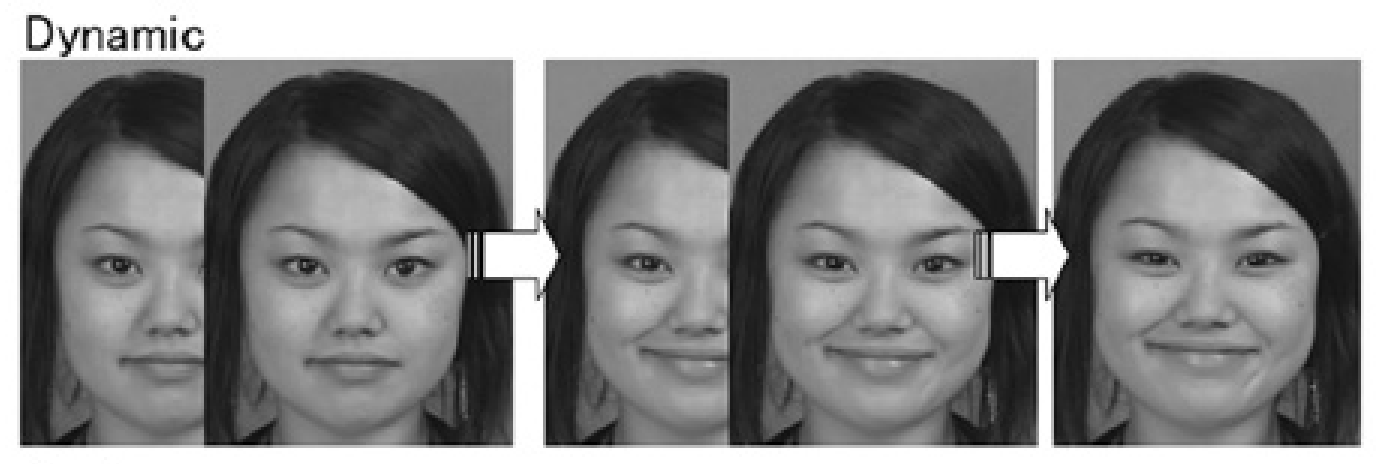

Static
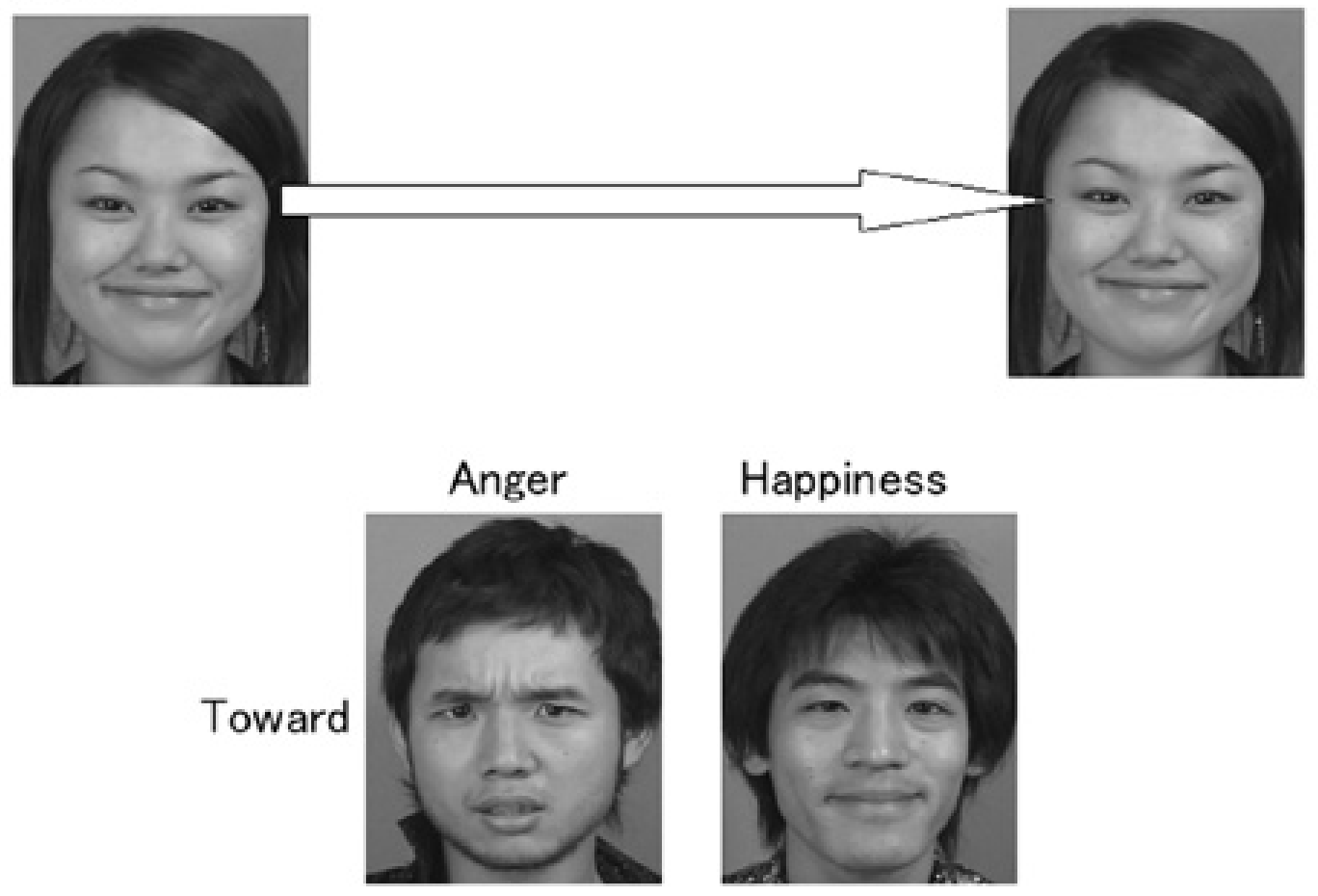

Away
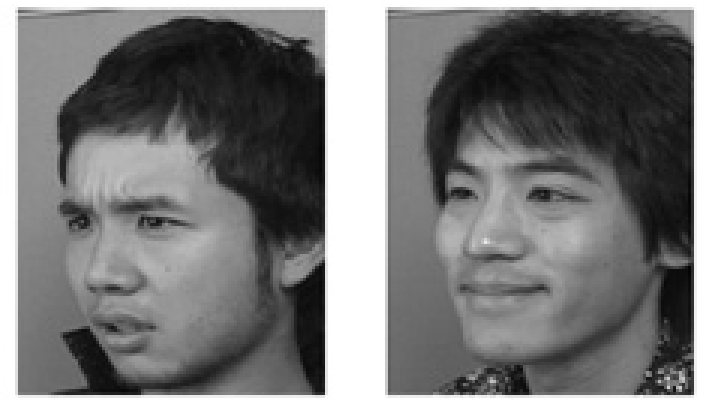

Fig. 1. Illustrations of stimulus presentations. (Upper) The sequence of stimulus presentation under dynamic and static presentation conditions. Under the dynamic condition, clips consisting of 38 frames ranging from neutral to emotional expressions were shown. Each frame was 
Neuroimage 32

shown for $40 \mathrm{~ms}$, and each clip was presented for $1520 \mathrm{~ms}$. Under the static condition, the frames showing the peak of expression in the dynamic condition were presented for $1520 \mathrm{~ms}$.

(Lower) Representative examples of angry and happy expressions

looking toward and away from the subject. The stimuli looking toward and away from the subject were recorded simultaneously by video cameras located in front of the model and at a $30^{\circ}$ angle to the model's left. 
Neuroimage 33
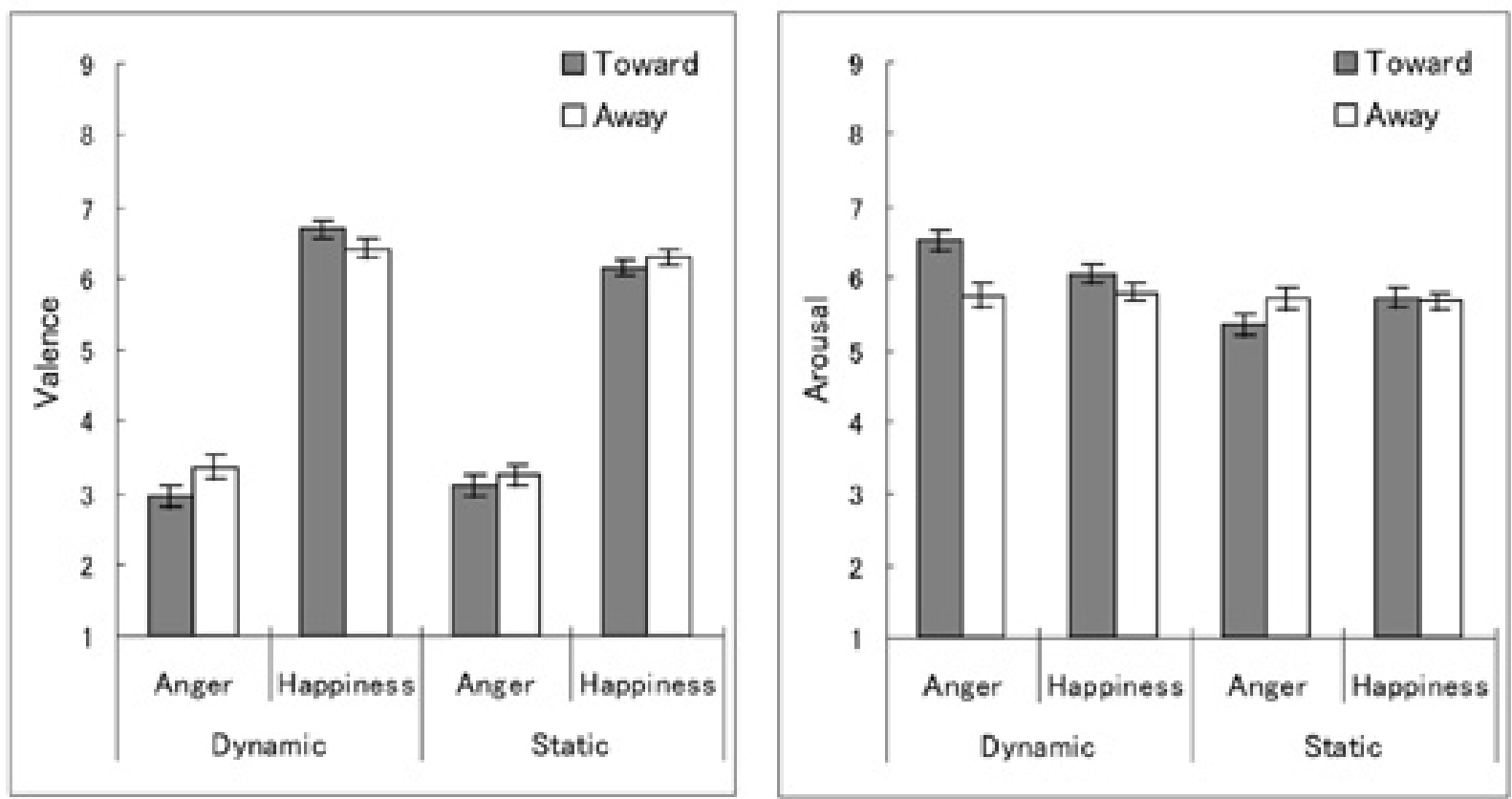

Fig. 2. Mean ( $\pm \mathrm{SE}$ ) ratings of experienced valence (left) and arousal (right). Note that a significant interaction between presentation condition and gaze direction was observed for arousal ratings, indicating that expressions looking toward the subject elicited greater arousal than expressions looking away from the subject under the dynamic presentation condition, but not under the static condition. 
Neuroimage 34
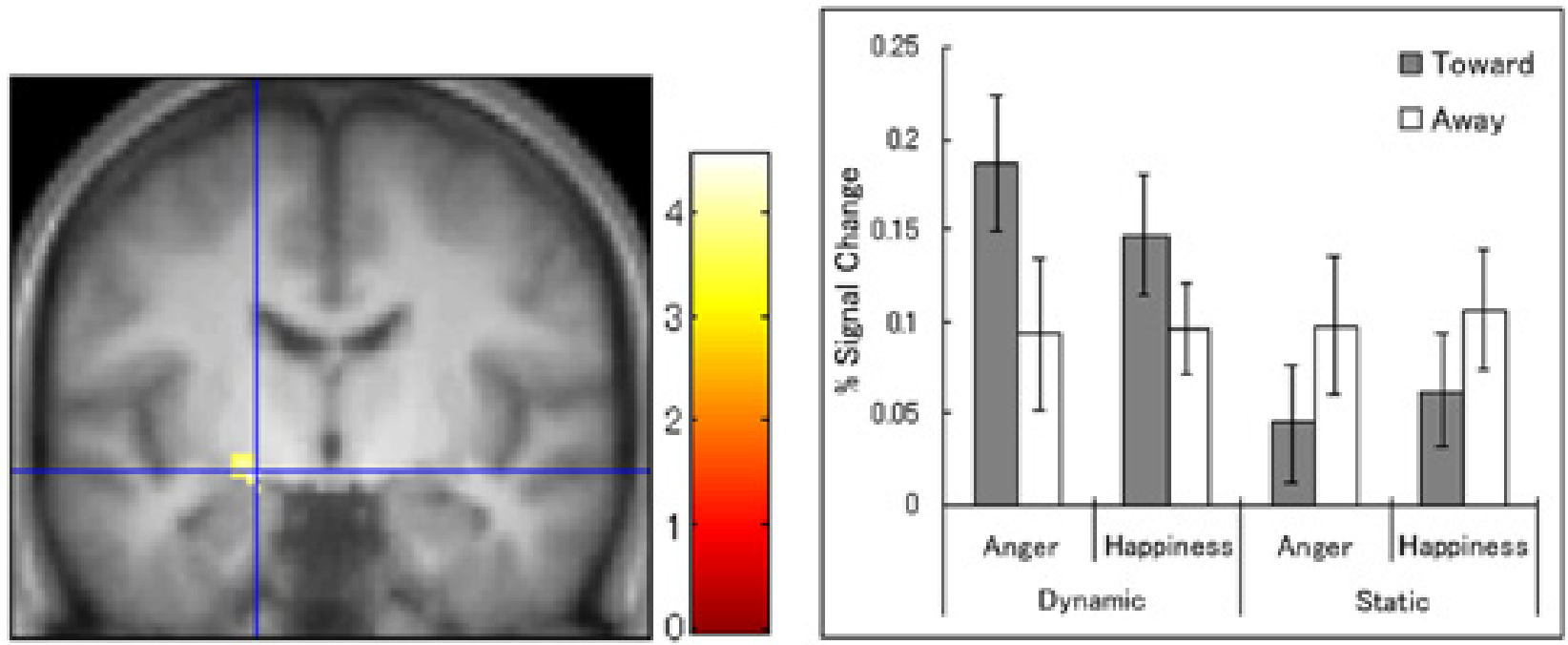

Fig. 3. The statistical parametric map (left) and mean $( \pm S E)$ percent signal changes showing the left amygdala activity for the interactions between presentation condition and gaze direction. The area is overlaid on the mean normalized structural MRI from all subjects in this study. Because the amygdala was our region of interest, a small volume correction was performed on the anatomically defined region. 